\title{
Empirical Potential Function for Simplified Protein Models: Combining Contact and Local Sequence-Structure Descriptors
}

\author{
Jinfeng Zhang ${ }^{1}$, Rong Chen ${ }^{1,2,3}$, and Jie Liang ${ }^{1}$ \\ ${ }^{1}$ Department of Bioengineering, \\ ${ }^{2}$ Department of Information \& Decision Science, \\ University of Illinois at Chicago, Chicago,IL, USA \\ ${ }^{3}$ Department of Business Statistics \& Econometrics, \\ Peking University, Beijing, P.R. China
}

October 30, 2018, Accepted by Proteins

\begin{abstract}
An effective potential function is critical for protein structure prediction and folding simulation. Simplified protein models such as those requiring only $C_{\alpha}$ or backbone atoms are attractive because they enable efficient search of the conformational space. We show residue specific reduced discrete state models can represent the backbone conformations of proteins with small RMSD values. However, no potential functions exist that are designed for such simplified protein models. In this study, we develop optimal potential functions by combining contact interaction descriptors and local sequence-structure descriptors. The form of the potential function is a weighted linear sum of all descriptors, and the optimal weight coefficients are obtained through optimization using both native and decoy structures. The performance of the potential function in test of discriminating native protein structures from decoys is evaluated using several benchmark decoy sets. Our potential function requiring only backbone atoms or $C_{\alpha}$ atoms have comparable or better performance than several residue-based potential functions that require additional coordinates of side chain centers or coordinates of all side chain atoms. By reducing the residue alphabets down to size 5 for local structure-sequence relationship, the performance of the potential function can be further improved. Our results also suggest that local sequence-structure correlation may play important role in reducing the entropic cost of protein folding.
\end{abstract}

Keywords: Decoy discrimination; discrete state model; potential function; protein structure prediction; simplified protein models; local sequence-structure relationship.

${ }^{*}$ Corresponding author. Phone: (312)355-1789, fax: (312)996-5921, email: jliang@uic.edu 


\section{Introduction}

Protein folding is a fundamental problem in molecular biology [1-3]. The thermodynamic hypothesis of protein folding postulates that the native state of a protein has lowest free energy under physiological conditions. Under this hypothesis, protein structure prediction, folding simulation, and protein design all depend on the use of a potential function. In protein structure prediction, the potential function is used either to guide the conformational search process, or to select a structure from a set of possible sampled candidate structures.

There are several challenging difficulties in computational studies of protein structures. The search space of protein conformation is enormous, and the native structure cannot be identified by exhaustive enumeration. This is the well-known "Levinthal's paradox" [4]. In addition, we do not yet have full understanding of all the physical factors and how they work collectively in folding proteins and maintaining protein stability. Simplified protein models provide an attractive approach that helps to overcome these two difficulties $[5,6]$. Based on simplified protein representation, these models can effectively reduce the complexity in conformational search. They are also valuable for isolating and identifying the most relevant factors contributing to protein folding, without the need to model an overwhelming amount of detailed atomistic information required when all-atom representation of protein structure is used.

There are several key technical issues in using simplified protein models. First, which form of the simplified protein representation would contain the needed relevant information? Second, what descriptors should we choose to extract the necessary information? Finally, how do we construct a potential function using these descriptors so near native structures will have lower energy than others? In this study, we develop an empirical potential function for simplified protein models at the residue-level. Our work fills an important gap. Existing empirical potential functions require either all-atom representation of protein structures [7-10], or the coordinates of the geometric center of side chains [11,12], which require explicit model of side chain atoms. Currently, there is no accurate potential function designed for simplified protein models requiring only $C_{\alpha}$ or backbone atoms. An effective potential function is essential for efficient conformational search, for evaluation of sampled structures, and for realization of the capabilities of a well-designed simplified protein model.

In this study, we choose the discrete state off-lattice model originally developed by Park and Levitt as the reduced representation for protein structures [13]. The states of this model for each residue is parameterized by a bond angle and a torsion angle. This model has been shown to work well in modeling protein structures, at the same time maintaining a low complexity [13]. We extend the original model and develop a set of optimal discrete states for each amino acids through clustering of the observed angles in native protein structures.

For these simplified off-lattice discrete state models, we follow a novel approach to develop descriptors. We use both two body residue contact interactions and the local sequence-structure information of two sequence nearest neighboring residues. Contact interactions capture several basic physical forces important for protein folding, including hydrophobic interactions, hydrogen bonding, charge interactions, and disulfide bonding interactions [14]. Contact interactions have been used in many empirical potentials $[7-12,15]$. The local sequence-structure correlation of residues capture the propensity of small sequences adopting specific local spatial structures. The existence of such propensity has been well recognized and it has been used in protein structure prediction $[16,17]$, in remote homology detection [18], and in discriminating native structures from decoys [19-22]. The non-overlapping nature of these two types of descriptors indicates that they contain different information. To our best knowledge, potential function developed in this study is the first to combine both types of descriptors.

There are two approaches for developing an empirical potential. One approach uses only native protein structures and apply statistical analysis to extract information important for protein stability $[7-9,11]$. The other approach uses both native protein structures and decoy conformations and apply optimization (or machine learning) techniques to derive a potential 
function that separates native structures from decoy structures [23-26]. The approach based on statistical analysis has the drawback of assuming explicitly or implicitly an unrealistic reference state such as random mixture model [11], and ignoring chain connectivity [27]. The approach based on optimization involves deriving parameters from a set of training proteins and decoys, and is attractive because it incorporates information contained in the decoy structures that are absent in native structures. The collection of a very large number of decoy structures plays the role of the reference state in the statistical methods. In addition, the optimization approach allows more flexibility in combining descriptors of different physical nature. To develop potential function by optimization, it is important to select a small or moderate number of descriptors to avoid over-fitting the training examples. For this purpose, we systematically develop several reduced alphabet of amino acid residues for both contact interactions and for local sequencestructure descriptors.

The potential function we developed here are tested for discrimination of native protein structures from several benchmark sets of decoy non-protein conformations. For all the decoys tested, the performance of our potential is comparable or better than several well-known residuelevel potential functions that requiring more complex protein representations. Our paper is organized as following: first, we introduce the simplified representation of protein structures. Second, we discuss the reduction of amino acid alphabet for neighboring interaction patterns. We then describe the descriptors and the form of the potential function, along with the optimization method to derive the weight vector of the potential function. This is followed by description of the performance of the potential function in discriminating native structures from decoys. Finally, we conclude this paper with discussion.

\section{Model and Methods}

\subsection{Representation of protein structures.}

Discrete state model. We use an off-lattice discrete state model to represent the protein structure [13]. In addition to $C_{\alpha}$ atoms, we use one additional atom $S C$ to model all side chain atoms, which is attached to the main chain $C_{\alpha}$ atoms, as shown in Figure 1. The distance between adjacent $C_{\alpha}$ atoms is fixed to $3.8 \AA$. The distances between $C_{\alpha}$ and side chain atoms, as well as the radius of each side chain atom depend on the residue type. Their values are taken from reference [28]. There is no additional increase in the degree of freedom due to the introduction of the side chain atom.

Similar to the fact that the position of $C_{\beta}$ atom of the side chain in a protein is uniquely determined from the positions of backbone atoms as the atoms connected to carbons have fixed angles, the position of the residue-dependent side chain atom $S C$ in this model is determined from backbone $C_{\alpha}$ atom [28].

There are 20 different types of atoms altogether (one $C_{\alpha}$ for backbone and glycine, and 19 different $S C$ atoms for different side chains). The backbone structure of a protein can be described by the bond angles $\alpha_{i}$ and torsion angles $\tau_{i}$ at the $i$-th $C_{\alpha}$ position (Figure 1). The overall three dimensional structure is completely determined by the set of angles $\left\{\left(\alpha_{i}, \tau_{i}\right)\right\}$ at each $C_{\alpha}$ position, except the terminal residues.

$\alpha$ and $\tau$ angles. To find the desirable number of states and the associated $(\alpha, \tau)$ values of amino acid residues for the discrete state model, we obtained the distribution of $\alpha$ and $\tau$ angles in 1,318 non-homologous X-ray protein structures from CulledPDB [29] (Figure 2), where the sequence identity between any pairs of proteins is less than 30 percent, and the resolution of the structures is better than $2 \AA$. Analogous to the Ramachandran plot, the distribution of $\alpha$ and $\tau$ angles also has densely and sparsely populated regions, which correspond to different secondary structure types The distribution of $(\alpha, \tau)$ angles differs for different amino acids.

Reduced discrete states. For each residue, we obtain a Cartesian coordinate system by taking the plane formed by $C_{i-2}, C_{i-1}$, and $C_{i}$ as the $x-y$ plane, and placing the origin at 


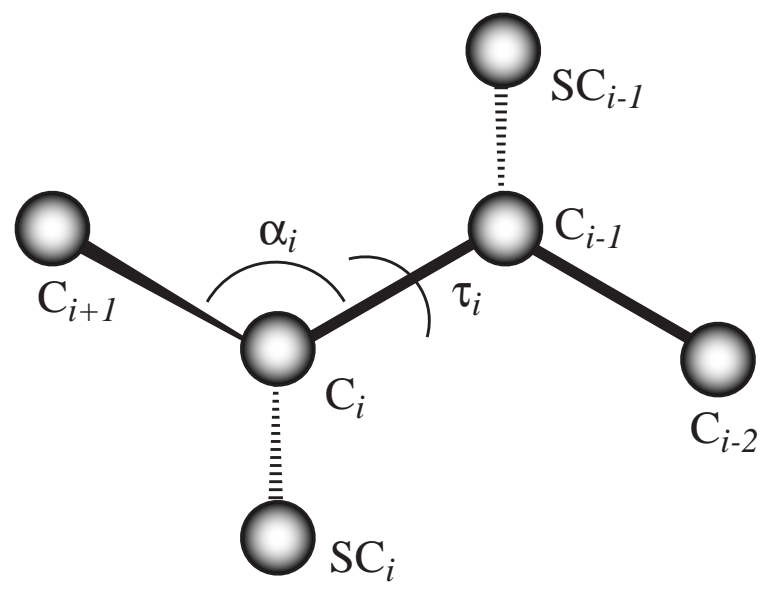

Figure 1: Discrete state model. $\alpha_{i}$ and $\tau_{i}$ angles are shown for residue $i$. Bond angle $\alpha_{i}$ at position $i$ is formed by $C_{i-1}, C_{i}$, and $C_{i+1}$. Torsion angle $\tau_{i}$ is the dihedral angle of the two planes formed by atoms $\left(C_{i-2}, C_{i-1}, C_{i}\right)$ and $\left(C_{i-1}, C_{i}, C_{i+1}\right)$.

$C_{i}$. The vector from $C_{i-1}$ to $C_{i}$ is taken as the direction of $x$-axis. After normalizing the bond length between $C_{i}$ and $C_{i+1}$ to unit length, we cluster the positions of $C_{i+1}$ atoms for all residues of the same type, which are taken as $C_{i}$. $k$-mean clustering is applied to group points on the unit sphere into $k$ (from 3 to 10) clusters for each amino acid residue type, where $k$ corresponds to the number of states for the amino acids. The centers of the clusters are then measured for the $\alpha$ and $\tau$ angles, which are taken as the optimized values of the discrete states of the amino acids. The values of discrete state angles for the 4 state model are listed in Table I. The values for $k$-state model of $k=5-10$ are listed in supplementary material.

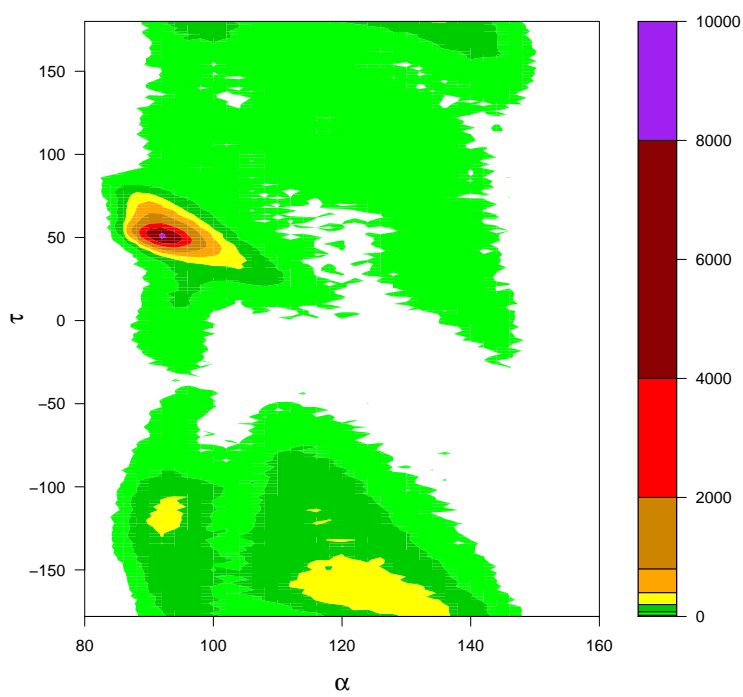

Figure 2: Distribution of $\alpha$ and $\tau$ angles taken by 20 natural amino acids in native proteins.

To study the effect of the preceding residue to the distribution of the discrete state of each 
Table I: Values of discrete state angles for 4 state model.

\begin{tabular}{|r|r|r|r|r|r|r|r|r|}
\hline A.A. & $\alpha_{1}$ & $\tau_{1}$ & $\alpha_{2}$ & $\tau_{2}$ & $\alpha_{3}$ & $\tau_{3}$ & $\alpha_{4}$ & $\tau_{4}$ \\
\hline $\mathrm{A}$ & 104.9 & -112.3 & 91.80 & 52.10 & 125.3 & -175.7 & 134.8 & 86.03 \\
$\mathrm{C}$ & 112.4 & -107.0 & 98.07 & 45.22 & 123.9 & -170.3 & 120.3 & 111.4 \\
$\mathrm{D}$ & 106.3 & -108.9 & 96.31 & 45.00 & 113.1 & -168.9 & 113.7 & 107.1 \\
$\mathrm{E}$ & 106.9 & -106.3 & 94.64 & 49.22 & 117.9 & -165.8 & 116.3 & 113.7 \\
$\mathrm{~F}$ & 112.2 & -105.9 & 98.50 & 46.15 & 122.9 & -166.7 & 120.9 & 116.0 \\
$\mathrm{G}$ & 108.2 & -96.99 & 102.3 & 36.01 & 124.9 & -165.0 & 133.1 & 110.4 \\
$\mathrm{H}$ & 108.3 & -101.6 & 98.73 & 45.03 & 119.6 & -164.7 & 122.9 & 112.8 \\
$\mathrm{I}$ & 110.3 & -108.5 & 95.57 & 47.44 & 119.2 & -163.9 & 116.4 & 115.4 \\
$\mathrm{~K}$ & 108.1 & -108.9 & 95.28 & 48.98 & 116.9 & -164.8 & 117.0 & 115.8 \\
$\mathrm{~L}$ & 110.3 & -110.7 & 94.31 & 48.84 & 117.7 & -163.9 & 115.3 & 114.6 \\
$\mathrm{M}$ & 110.8 & -107.1 & 94.50 & 49.24 & 121.7 & -166.0 & 118.8 & 116.6 \\
$\mathrm{~N}$ & 106.2 & -109.6 & 96.27 & 41.75 & 116.9 & -172.9 & 122.3 & 99.00 \\
$\mathrm{P}$ & 110.1 & -104.3 & 93.65 & 41.43 & 105.0 & -163.4 & 100.0 & 131.7 \\
$\mathrm{Q}$ & 108.4 & -109.7 & 94.70 & 49.15 & 119.3 & -167.2 & 117.8 & 112.1 \\
$\mathrm{R}$ & 108.4 & -112.9 & 93.20 & 49.67 & 121.4 & -174.3 & 127.6 & 93.57 \\
$\mathrm{~S}$ & 114.5 & -103.3 & 99.38 & 49.30 & 120.8 & -163.8 & 119.0 & 122.1 \\
$\mathrm{~T}$ & 115.5 & -105.7 & 99.57 & 47.03 & 121.6 & -165.2 & 121.2 & 122.0 \\
$\mathrm{~V}$ & 111.1 & -110.3 & 96.96 & 46.87 & 121.0 & -165.1 & 117.5 & 116.4 \\
$\mathrm{~W}$ & 112.4 & -105.3 & 96.64 & 48.12 & 121.5 & -166.4 & 117.7 & 119.9 \\
$\mathrm{Y}$ & 113.3 & -103.5 & 99.41 & 45.47 & 124.2 & -166.5 & 119.5 & 118.3 \\
\hline
\end{tabular}

type of amino acids, we plotted the conditional distribution of the discrete state for each amino acid residue given the state of the preceding residue. The results for alanine is shown in Figure 3a, which shows that the distribution of the discrete state of alanine is affected significantly by the state of the preceding residue. This distribution is also affected by the type of preceding residue, as shown in Figure 3b. Similar effects are observed in all other residues.

Mapping of X-ray structures to discrete state models. The conformational space associated with a discrete state representation is different from the continuous conformational space of a protein structure in $\mathbb{R}^{3}$. To represent a protein in the simplified discrete space, we need to map a protein structure from the continuous conformational space to a structure in a discrete space, with the requirement that it must be the one most similar to the real protein structure among all possible structures in the simplified conformational space by some similarity measure. In this study, we use both global structural similarity and local structural similarity criteria. To generate globally similar discrete structures to an X-ray structures, we use a heuristic "build-up" algorithm first introduced by Park and Levitt [13]. In this method, the protein structure is constructed in single residue increments starting from the $\mathrm{N}$-terminus. At each step of construction, only a fixed number of $m$ structures with the lowest RMSD from the partial X-ray structure are retained. When a residue is added to the growing chain, all $k$ possible states on each of the retained chains are examined for conformation similarity to X-ray structure. This gives $k \times m$ possible conformations at each step for a $k$-state model, of which the best $m$ conformations are retained for the next step of construction. The representatives obtained from the build-up method are the ones among the final $m$ full protein candidate structures that has the lowest global RMSD values from the native structure. With this method, we obtained $m=5,000$ discrete structures for each proteins in the set of 70 representative proteins obtained in [31], with average length of 136 residues for 3 to 10 discrete states. The average RMSD values of the best fitted structures for each discrete state are shown in Figure 4. We also fitted 978 proteins with less than 500 residues taken from the 1,318 proteins in the CulledPDB with $m=2,000$ conformations for each proteins for state 3 to 6 and obtained similar results with slightly larger average RMSD values. In general, the average RMSD values of the best models to the native structures is about $2.3 \AA$ for 4 -state model, $1.9 \AA$ for 5 -state model, $1.6 \AA$ for 

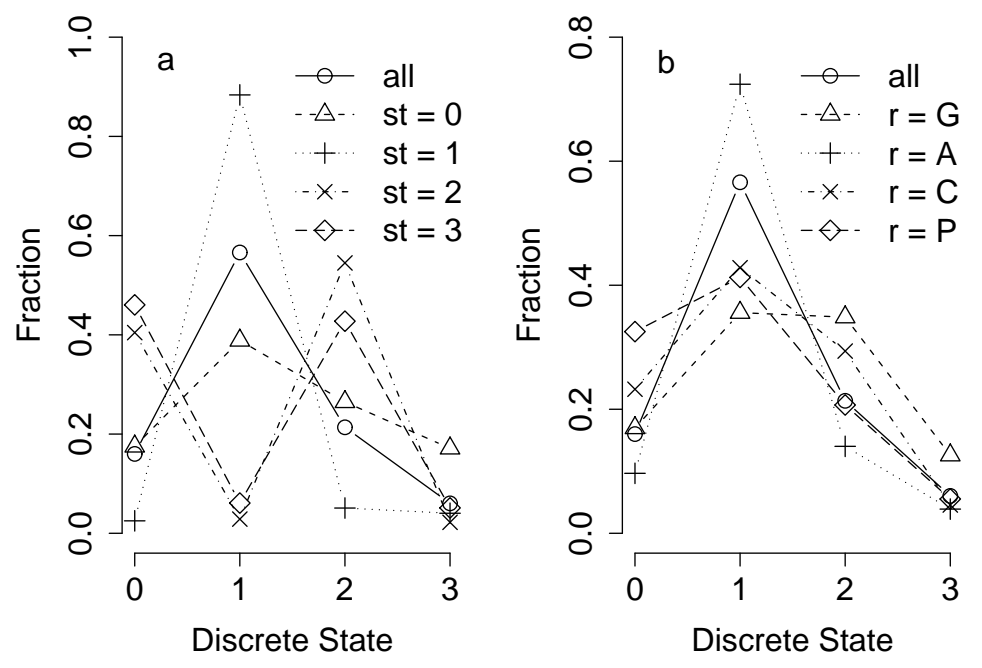

Figure 3: The distribution of discrete states of alanine calculated from 1,318 non-homologous $\mathrm{x}$-ray protein structures given (a) the discrete state or (b) the type of preceding residue. $Y$-axis shows the fraction of different discrete state of alanine as labeled on $\mathrm{X}$-axis. Labels in legend: all, marginal distribution of discrete state of alanine regardless the state or residue type of the preceding residue; st, the discrete state of the preceding residue; $r$, the type of the preceding residue. We applied a bootstrap procedure using 1,000 sampling with replacement following [30] to obtain the confidence intervals for the data shown. The $95 \%$ confidence intervals for the above data are all within $(f-0.03, f+0.03)$, where $f$ is the fraction shown on the figure.

6-state model, and near $1.0 \AA$ for 10 -state model.

The high quality of the discrete state models when fitted to X-ray structures indicates that a model with four to six states is sufficient to generate near native structures with low RMSD values, i.e., $<3 \AA$ to native structures. In the rest of the paper, we use 4 -state model for its simplicity.

To generate discrete state model by local structural similarity, each residue is simply assigned a discrete state that is most similar to its local $(\alpha, \tau)$ angle in X-ray structure. The resulting structure has maximum local similarity to X-ray structures [22].

\subsection{Descriptors.}

Simplified amino acid alphabet $\Sigma_{1}$ by neighbor interactions. Early protein synthesis has been thought to involve a reduced amino acid alphabet [32]. Previous work has shown that a small $\beta$-sheet protein, the SH3 domain, can be encoded by a reduced 5 -letter amino acid alphabet [32]. Despite the dramatic changes in sequence, the folding rates of the protein encoded by the reduced alphabet are very close to that of the naturally occurring SH3 domain [32]. Various reduced amino acid alphabets have been obtained previously based on the analysis of amino acid substitution matrix, contact propensity, or information theory [15,32-36]. The resulting reduced amino acid alphabets are useful in protein folding studies and in identifying consensus sequences from multiple alignment $[37,38]$. However, there has been no attempt to derive reduced alphabet based on the local sequence-structure relationship of the amino acids. By recognizing strong similarities of different amino acids in their local spatial interaction patterns, 


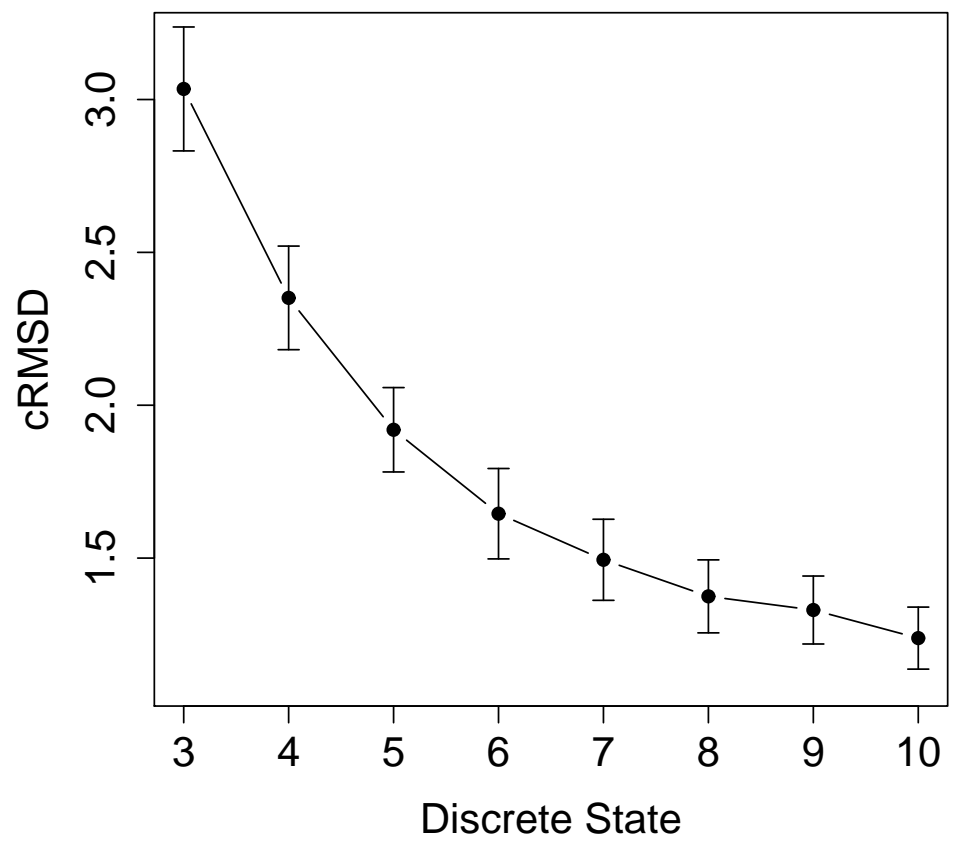

Figure 4: Average RMSD values of the best 2,000 discrete state models $v s$. the number of states. The average and standard deviation of RMSD values are calculated from a set of 70 proteins obtained from [31]. The average length of these proteins are 137.

one can summarize the relationship between local sequence and structure more succinctly and accurately. In addition, using reduced alphabet also alleviates problems arising from the use of a limited data set of protein structures and decoys to derive empirical potential functions. Such a simplified alphabet would also be useful in representation of protein structures, in building fragment libraries, in prediction of local structures from local sequences, and in generating protein like conformations using chain growth method [39-43].

Different amino acids have different distributions of $\alpha$ and $\tau$ angles. However, amino acids that are similar in geometrical shapes or chemical properties often share similar patterns in the distribution of $\alpha$ and $\tau$ angles. The posterior distribution of discrete state angles for a residue given the preceding residue's type and discrete state provides characteristic information of local structure of residues. The observed neighboring residue effect shown in Figure 3 indicates that the type and geometry represented by discrete state of one residue also affect the geometry of its adjacent residues. These observations prompt us to simplify the twenty amino acids alphabet to a smaller alphabet. To derive the simplified alphabet, we estimate the first order state transition probability of residues in native protein structures as described below.

A protein structure can be represented uniquely by a sequence of $(a, x)$, where $a$ is amino acid residue type and $x$ is the discrete state. For a four-state model with 20 amino acid types, the total number of possible descriptors for one residue position is $20 \times 4=80$. For simplicity, we use $s \in[1 \ldots 80]$ to represent the state a residue may take, i.e., the discrete conformational state and the amino acid type. We define the first order state transition probability $p_{s_{1}, s_{2}}$ as: $p_{s_{1}, s_{2}}=$ $p\left[s_{2} \mid s_{1}\right]=p\left[\left(a_{2}, x_{2}\right) \mid\left(a_{1}, x_{1}\right)\right]$ and calculate the transition matrix from 1,318 non-homologous proteins. We then cluster different residue types based on the transition probabilities. Each 


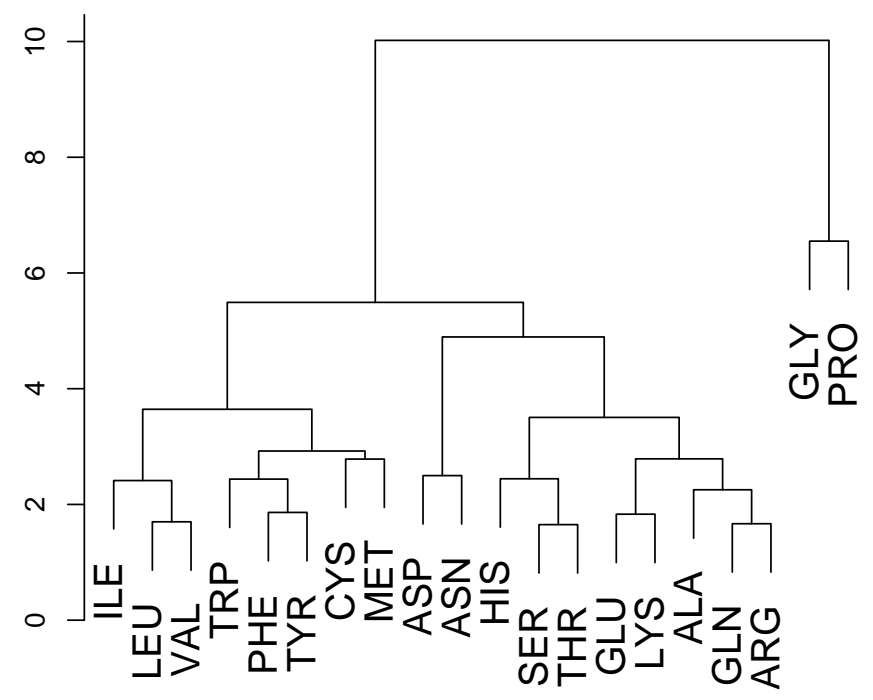

Figure 5: Hierarchical clustering of amino acids using their neighboring residue interaction patterns. Glycine and proline are residues having highest tendency of being at turn and loop positions, which are separated from the rest of amino acids. The rest 18 amino acids are clustered into two groups roughly according to their hydrophobicities.

type of residue corresponds to a vector of 320 transition probabilities (moving from one of the four states associated with this particular type of residue to one of the 80 different residue type and state combinations). We define the distance between two amino acid types as the Euclidean distance between the corresponding transition probabilities vectors. Results of clustering of amino acids using this distance metric are shown in Figure 5. The twenty amino acids can be divided into two distinct groups, with one group containing only glycine and proline. Our clustering results are very different from those using other criteria. Geometrically, it is intuitive that glycine and proline have no side chains and are different from other residues. For this study, we further group the residues into an alphabet $\Sigma_{1}$ of 5 letters with $\mathcal{A}=\{A, E, K, Q, R, S, H, T\}$, $\mathcal{B}=\{C, I, V, L, M, W, F, Y\}, \mathcal{C}=\{D, N\}, \mathcal{D}=\{G\}$, and $\mathcal{E}=\{P\}$.

Simplified amino acid alphabet $\Sigma_{2}$ for contact propensity: incorporating additional descriptors. We use a different reduced alphabet for contact interactions. In this work, we take an alphabet $\Sigma_{2}$ based on results published in [15], where an alphabet of ten residue types are chosen as following: $\{I, L, V\},\{C\},\{A\},\{G\},\{N, Q, S, T\},\{P, H\},\{M, F\},\{W, Y\}$, $\{D, E\},\{K, R\}$. This reduced alphabet is used for simplification of contact descriptors.

Descriptor set $\mathcal{D}_{1}$. To encode the information contained in local-sequence and localstructure of sequence neighboring residues, we use the two discrete state taken by two consecutive residues, $\left(x_{i-1}, x_{i}, a_{i-1}, a_{i}\right)$ as descriptors. The number of possible descriptor values for a pair of residues is $(4 \times 4) \times(5 \times 5)=400$, since there are 4 discrete conformational states and 5 simplified amino acid types in alphabet $\Sigma_{1}$.

Descriptor set $\mathcal{D}_{2}$. Contact interactions in a protein structure can be uniquely defined 
once the contact criterion is given. With 20 types of atoms (19 side chain atoms and 1 backbone atom), the number of different types of contacts, or contact descriptors is 210 . This set of descriptors is denoted as $\mathcal{D}_{21}$. When using 10 reduced atom types derived from the simplified amino acid alphabet $\Sigma_{2}$ with 10 amino acid types, the number of contact descriptors is 55 , and we denoted it as $\mathcal{D}_{2,2}$. Because of the reduction in the number of descriptors, we can afford to incorporate additional descriptors that are more informative. As an exploratory study, we further distinguish each pairwise contact type by the sequence separation $d_{i, j}$ of the two contacting residues, where $d_{i, j}=|j-i|$. We group $d_{i, j}$ values into three bins, with bin 1 for $d_{i, j}=4$, bin 2 for $d_{i, j}=5$, and bin 3 for $d_{i, j}>5$. Thus, the total number of contact descriptors becomes $55 \times 3=165$. This set is denoted as $\mathcal{D}_{2,3}$.

Combining contact and local sequence-structure descriptors. We have experimented with three different sets of descriptors obtained by combining 400 local sequencestructure descriptors $\mathcal{D}_{1}$ with the three different sets of contact descriptors $\mathcal{D}_{2,1}, \mathcal{D}_{2,2}$ and $\mathcal{D}_{2,3}$. CALS (Contact And Local Sequence-structure) is the set of 610 descriptors combining $\mathcal{D}_{1}$ and $\mathcal{D}_{2,1}$. RCALS1 (Reduced Contact And Local Sequence-structure 1) is the set of 455 descriptors combining $\mathcal{D}_{1}$ and $\mathcal{D}_{2,2}$. RCALS2 (Reduced Contact And Local Sequence-structure 2) is the set of 565 descriptors combining $\mathcal{D}_{1}$ and $\mathcal{D}_{2,3}$.

We also study the property of using contact descriptor $\mathcal{D}_{1}$ only (denoted as $\mathrm{C}$ potential) and local sequence-structure descriptors $\mathcal{D}_{21}$ only (denoted as LS potential).

Calculation of the contact descriptors from a structure. If all backbone atoms are present in a given structure, we obtain each $S C$ atom position by extending the bond length between $C_{\alpha}$ and $C_{\beta}$ atoms to a residue dependent value along a fixed direction as in [28], and the new position of the $C_{\beta}$ is taken as the position of side chain atom $S C$. If only $C_{\alpha}$ atoms is present, we estimate the $S C$ position for side chain atom following the approach of [28], where the coordinates of side chain atom at position $i$ is approximately determined by the coordinates of $C_{\alpha}$ s at position $i-1, i$, and $i+1$. After all $C_{\alpha}$ and $C_{\beta}$ atoms have been placed, we calculate the contact descriptors by simply measuring the pair-wise distance of atoms. In our calculations, explicit information from side chain atoms of a PDB structure is never used.

To derive local sequence-structure descriptors, we transform the structure to a discrete state model using local fit as described earlier. The local sequence-structure descriptors are calculated directly from the discrete representation.

\subsection{Empirical potential function.}

Potential functions based on physical model (such as CHARMM and AMBER [44, 45]) require allatom representations of protein structures to model detailed physical forces and therefore are inappropriate for simplified protein representations. With a proper representations of protein sequence and structure, and a set of descriptors specified, we have a description function, $c=$ $f(\boldsymbol{s}, \boldsymbol{a})$, which takes a pair of structure $\boldsymbol{s}$ and sequence $\boldsymbol{a}(\boldsymbol{s}, \boldsymbol{a})$, and maps it to a descriptor vector $\boldsymbol{c}$. The next step is to decide on the form of the potential function $E=H(\boldsymbol{c})$, which maps the vector $\boldsymbol{c}$ to a real valued energy or score, $E$.

The form of a potential function in this study is a linear combination of the descriptors: $H(\boldsymbol{c})=\boldsymbol{w} \cdot \boldsymbol{c}$, i.e., the inner product of the descriptor vector $\boldsymbol{c}$ and the weight vector $\boldsymbol{w}$. The energy landscape of an empirical potential function defined for simplified protein model is inevitably different from the true energy landscape of a real protein. For tasks such as protein structure prediction, the minimum requirement is that the structures in the conformational space of simplified protein model that are closest to the native structure have the globally minimum energy values. Developing such a potential function is challenging, as it is not even known that whether near native conformations in simplified protein representation can be the most stable conformations in the full conformational space under any particular potential functions [46]. Despite this uncertainty, there is still a great deal of interest and work in developing optimized potential functions that stabilizes native proteins in simplified protein models $[26,47-51]$. This 
work is a continuation of efforts in this direction.

We obtain weight vector $\boldsymbol{w}$ using optimization method [26,47-51]. For our linear potential functions, the basic requirement is: $\boldsymbol{w} \cdot\left(\boldsymbol{c}_{N}-\boldsymbol{c}_{D}\right)+b<0$, where $\boldsymbol{c}_{N}$ and $\boldsymbol{c}_{D}$ are the native descriptor vector and the decoy descriptor vector for one protein, and $b \geq 0$ is the energy gap between a native and decoy structure that should exists. Each pair of native vector and decoy vector serves as one inequality constraint. All of the constraints jointly define a convex polyhedron $P$ for feasible weight vectors $\boldsymbol{w}$ 's. If $P$ is not empty, there could be an infinite number of choices of $\boldsymbol{w}$, all with perfect discrimination [26]. To find a weight vector $\boldsymbol{w}$ that is optimal, one can choose the weight vector $\boldsymbol{w}$ that minimizes the variance of score gaps between decoys and natives [47], or minimizing the $Z$-score of the native protein and an ensemble of decoys $[48,49]$, or maximizing the ratio $R$ between the width of the distribution of the score and the average score difference between the native state and the unfolded ones [52]. Previous works using perception learning and other optimization techniques $[47,50-53]$ showed that often effective linear sum potential functions can be obtained.

Here we obtain the optimal weight vector $\boldsymbol{w}$ by solving the following primal quadratic programming problem:

$$
\begin{aligned}
& \text { Minimize } \quad \frac{1}{2}\|\boldsymbol{w}\|^{2} \\
& \text { subject to } \boldsymbol{w} \cdot\left(\boldsymbol{c}_{N}-\boldsymbol{c}_{D}\right)+b<0 \text { for all } N \in \mathcal{N} \text { and } D \in \mathcal{D} \text {. }
\end{aligned}
$$

The solution maximizes the distance $b /\|\boldsymbol{w}\|$ of the plane $(\boldsymbol{w}, b)$ to the origin [54]. We use a support vector machines (SVM) for this task [55].

Potential function studied. Based on the five different sets of descriptors described above, we study the following five different potential functions: CALSP (potential function based on the CALS descriptor set), RCALSP1 (potential function based on the RCALS 1 descriptor set), RCALSP2 (potential function based on the RCALS 2 descriptor set), CP (potential function based on the C descriptor set), and LSP (potential function based on the LS descriptor set).

\subsection{Data set for discrimination test.}

Proteins database. We select 978 non-homologous proteins from CulledPdb [29], with the criteria that the sequence identity is less than $30 \%$, the resolution of X-ray structures is smaller than $2 \AA$, and the $\mathrm{R}$ factor is smaller than 0.25 . In addition, using a compactness parameter $z_{\alpha}$ developed in [42], we require that all have $z_{\alpha}$ values greater than 3.0, so the compactness of the protein is that of the single domain globular proteins. This compactness constraint excludes proteins with extended conformations. These proteins are unlikely to be stable on their own, and usually requires protein-protein interactions or protein-DNA interactions [10,26].

Gapless threading decoys. We use gapless threading to generate a total of about 60 millions of decoys [56]. A three fold cross validation is applied to train the potential function and test its performance.

Decoys generated by Loose et. al. (LKF decoy set). This set of decoys are generated by Loose, Klepeis, and Floudas using the program of DYANA, which takes as input the sequence of a protein, along with information about its secondary structure that gives bounds for the distances and torsion angles between atoms $[57,58]$. DYANA minimizes the energy of the structure and then simulates a sharp increase in temperature, with a step using molecular dynamics simulation that allows the shape of the protein to change. The protein is then slowly cooled down, or annealed, and its energy is again minimized to give the output structure. Decoys for 185 proteins were downloaded from the authors' website. About 200 decoy structures for each protein are available to us [57].

Decoys generated by Baker et. al. (Baker decoy set). This set of decoys has 41 proteins. All decoys are generated by the program Rosetta [59]. Several different protocols are combined to produce the decoy set, which has the following properties: (1) It contains conformations for a wide variety of different proteins; (2) it contains conformations close $(<4$ 
$\AA$ ) to the native structure; (3) it consists of conformations that are at least near local minima for a reasonable potential function, so they cannot be trivially excluded based on obviously non protein like features; and (4) it is produced by a relatively unbiased procedure that does not use information from the native structure during conformational search [60].

4State_reduced set. This decoy test set contains native and near-native conformations of seven sequences, along with about 650 misfolded structures for each sequence. Park and Levitt generated the positions of $C_{\alpha}$ in these decoys by exhaustively enumerating 10 selectively chosen residues in each protein using a 4-state off-lattice model. All other residues were assigned the $\phi / \psi$ values based on the best fit of a 4 -state model to the native chain. Conformations in the decoy sets all have low scores by a variety of potential functions, and low root-mean square distance (RMSDs) to the native structures [61].

Lattice_ssfit set. The Lattice_ssfit set contains conformations for eight small proteins generated by ab initio protein structure prediction methods. The conformational space of a sequence was exhaustively enumerated on a tetrahedral lattice. A lattice-based potential function was used to select the 10,000 best-scoring conformations. Park and Levitt fitted secondary structures to these conformations using a 4-state model. The 10,000 conformations were further scored with a combination of an all-atom potential function, a hydrophobic compactness function, and a one-point per residue potential function. The 2,000 best-scoring conformations for each protein were selected as decoys for this data set $[62,63]$.

LMDS set. The local minima decoy set (LMDS) contains decoys derived from the experimentally obtained secondary structures of 10 small proteins belonging to diverse structural classes. Each decoy is a local minimum of a "hand-made" energy function. The authors generated ten thousand initial conformations for each protein by randomizing the torsion angles of the loop region [64]. The adjacent local minima were found by truncated Newton-Raphson minimization in torsion space. Each protein is represented in the decoy set by its 500 lowest energy local minima.

\section{Results}

\subsection{Performance on gapless threading decoys.}

The performance of the potential function on decoys generated by gapless threading is listed in Table II. A three-fold cross validation is employed to test the potential function, where all of the 978 proteins are randomly divided into three groups, and two groups and their associated decoys are used in turn for training and one group for testing. Among the 978 proteins, CALSP (see above) has only 6 proteins misclassified, which corresponds to an accuracy of 99\%. A protein is misclassified if there is one or more decoy structure(s) for that protein with a lower score than that of the native structure. The potential functions RCALSP1 and RCALSP2 also give good performance, with only 5 and 3 proteins misclassified, respectively. We also tested potential functions containing only contact (CP) and only local sequence-structure (LSP) descriptors. Clearly, combining both type of information in CALSP, RCALSP1, and RCALSP2 is much better than using CP or LSP alone. By comparing the performance of CP and LSP, we can also see that contact descriptors are more informative in discriminating native structures from decoys than local sequence-structure descriptors.

We also compare our potential functions with several other residue based potential functions, including those developed by Tobi et.al. (TE13) [12], Miyazawa \& Jernigan (MJ) [11], and Bastolla et. al. (BV) [10]. Although these potential functions are residue based potential functions, they need all-atom representation since they either need to calculate the side chain geometric centers or need to compute explicit atom-atom contacts. CALSP, RCALSP1, and RCALSP2 are the only potential functions, that can be applied on representations with only $C_{\alpha}$ and $C_{\beta}$ atoms. Since $C_{\beta}$ position is completely determined by coordinates of backbone atoms, these potential functions also work for representation with only backbone atoms. The results for 
Table II: Performance of residue based potential functions in decoy discrimination. The number of misclassifications are listed. CALSP: Contact And Local Sequence-structure Potential; RCALSP1: Reduced Contact And Local Sequence-structure Potential with 455 descriptors; RCALSP2: Reduced Contact And Local Sequence-structure Potential incorporating contact order information with 565 descriptors; CP: Contact potential using only contact component of CALSP; LSP: Local sequence-structure potential using only local sequence-structure component of CALSP; TE13: potential function developed by Tobi \& Elber [12]; BV: Potential function developed by Bastolla \& Vendruscolo [10]; MJ: Potential function developed by Miyazawa \& Jernigan [11]. AB: Computation of potential function needs only $C_{\alpha}$ and $C_{\beta}$ atoms; SCC: Computation of potential function needs side chain center; AA: Computation of potential function needs all-atom representation.

\begin{tabular}{|l|r|r|}
\hline Potential Function & Complexity & Mis-classified Proteins \\
\hline CALSP & $\mathrm{AB}$ & $6 / 978$ \\
\hline RCALSP1 & $\mathrm{AB}$ & $5 / 978$ \\
\hline RCALSP2 & $\mathrm{AB}$ & $3 / 978$ \\
\hline CP & $\mathrm{AB}$ & $24 / 978$ \\
\hline LSP & $\mathrm{AB}$ & $249 / 978$ \\
\hline \hline TE13 & SCC & $7 / 194$ \\
\hline BV & $\mathrm{AA}$ & $2 / 194$ \\
\hline MJ & SCC & $85 / 194$ \\
\hline
\end{tabular}

other potential functions are obtained from tables in [26], where the authors followed the original literature of contact definition, cut-off values, as well as using the original potential parameters. The training data and test data in [26] were obtained from the WhatiF database [65], while the set of 978 proteins are obtained from the CULLPDB dataset. Although direct comparisons using exactly the same set of proteins is impossible, the results listed in Table II indicate that despite using a much simplified representation, CALSP has comparable or better performance than other residue level potential functions requiring more detailed representations.

\subsection{Performance on other decoy sets.}

LKF Set. When the potential function obtained from training using gapless threading decoys is tested on other decoy sets, the performance of discrimination is rather poor. This is not surprising, since it is well known that gapless threading decoys are less challenging than explicit decoys generated by different energy minimization protocols. Potential functions derived by optimization frequently use more realistic decoys. We therefore develop a new version of potential function CALSP based on training with explicitly generated decoy conformations. The LKF and Baker decoy sets are used since these are the only ones with relatively large number of proteins and decoys (185 protein, 36,840 decoys for LKF decoy set, and 41 proteins, 76,224 decoys for Baker decoy set, respectively).

We use a four fold cross validation for the LKF decoy set, where all of the 185 proteins are randomly divided into four groups, and three groups are used in turn for training and one group for testing. No gapless threading decoys are included in training. As a comparison, the performance of the original LKF potential on 151 of the 185 proteins are listed in [57]. For these 151 proteins, 140 proteins collected from test sets of different cross validations are ranked as number 1 by our CALSP potential function with an average z-score of 6.42 , and 137 proteins ranked as number 1 by RCALSP1 with an average $z$-score of 6.15 . As a comparison, potential function LKF has 93 protein ranked as number 1 as reported in [57], with an average z-score of 3.08. Potential function TE13 has 64 protein ranked as number 1 as reported in [57], with an 
Table III: The number of mis-classifications using CALSP and other residue based potential functions. CALSP: Contact And Local Sequence-structure Potential; RCALSP1: Reduced Contact And Local Sequence-structure Potential with 455 descriptors; TE13: potential function developed by Tobi \& Elber; LKF: potential function developed by Loose, Klepeis, and Floudas.

$* z$-score is defined as $\left(\bar{E}-E_{n}\right) / \sigma$, where $\bar{E}$ is the average score of the decoys for a protein, $E_{n}$ is the score of native conformation, and $\sigma$ is the standard deviation of the scores of decoys.

** Result obtained from [57]. It is not trained on LKF decoy set.

\begin{tabular}{|l|r|r|}
\hline Potential Function & Mis-classified Proteins & $z$-score \\
\hline LKF decoy set & $11 / 151$ & 6.42 \\
\hline CALSP & $14 / 151$ & 6.15 \\
\hline RCALSP1 & $58 / 151$ & 3.08 \\
\hline LKF & $87 / 151$ & 2.01 \\
\hline TE13** & $13 / 41$ & 4.16 \\
\hline Baker decoy set & \multicolumn{3}{|c|}{} \\
\hline CALSP & \multicolumn{3}{|c|}{} \\
\hline
\end{tabular}

average z-score of 2.01 (Table III).

Because the Baker decoy set contains only 41 proteins, which is too small for a 4 -fold cross validation test, we carried out leave-one-out tests, again without including any gapless threading decoys during training. Even though only 40 proteins are available for training each time, our results are encouraging: we have 28 proteins ranked number 1 , with an average z-score of 4.16 (Table III).

For both LKF decoy sets and the Baker decoy set, we found that inclusion of gapless threading decoys does not offer significant improvement in performance. As discussed in [26], this is because only a small number of training examples will contribute as to determine the boundary between proteins and decoys. In the study of LKF and Baker decoys, few such training examples come from gapless threading decoys when the combined training sets are used. This confirms earlier observation that decoys from gapless threading are indeed less challenging. High quality decoys are very much in need for the development of potential functions by optimization methods.

Other Decoy Sets: 4State-reduced, LMDS, and Lattice-ssfit. We also test the CALSP potential function using the 4STATE-REDUCED decoy set, LMDS decoy set, and LATTICE_SSFIT decoy set. Because the number of proteins in these decoy sets are relatively small, we combined several training sets, including gapless threading decoys, the near native structures produced by the greedy build-up method and decoys from LKF decoy set. Performance of CALSP on these decoy sets is listed in Table IV. We compare CALSP with three other residuebased potential functions, namely, TE13, LL [15], and MJ. Performance of CALSP in general is better or comparable to other potential functions. It performs better than other potential functions on LMDS decoy set. Again, although all potential functions are of residue level, only CALSP can be applied to simplified models represented by $C_{\alpha}$ and $C_{\beta}$ atoms, or by backbone atoms only.

\section{Conclusion and Discussion}

Discrete state representation. In this study, we aim to develop an effective potential function for simplified protein models. We use discrete state model for representation of protein structures. We obtained discrete state values of bond angle $\alpha$ and torsion angle $\tau$ from 3 to 10 states (see supplementary data). By generating near native structures of low RMSD to native 
Table IV: Performance of CALSP for three decoy sets $[28,62,63,66]$. The numbers are the ranking of the native proteins. Results not available from the references are labeled as "N/A".

\begin{tabular}{|l|r|r|r|r|}
\hline Decoy sets & CALSP & LL 8 & TE13 & MJ \\
\hline A) 4state 28] & & & & \\
1ctf & 1 & 1 & 1 & 1 \\
1r69 & 1 & 1 & 1 & 1 \\
1sn3 & 2 & 1 & 6 & 2 \\
2cro & 2 & 1 & 1 & 1 \\
3icb & 1 & 5 & N/A & N/A \\
4pti & 2 & 1 & 7 & 3 \\
4rxn & 3 & 51 & 16 & 1 \\
\hline B) LMDS 66] & 1 & & & \\
1b0n-B & 436 & 217 & N/A & N/A \\
1bba & 1 & 1 & 1 & N/A \\
1ctf & 83 & 500 & 14 & 501 \\
1fc2 & 1 & 2 & 5 & 13 \\
1dtk & 1 & 9 & 2 & 1 \\
1igd & 3 & 17 & 1 & 11 \\
1shf-A & 1 & 1 & 1 & 1 \\
2ovo & 4 & 3 & 1 & N/A \\
4pti & 1 & 9 & N/A \\
\hline C) lattice_ssfit $[62,63]$ & & & & \\
1beo & 1 & 1 & N/A & N/A \\
1ctf & 1 & 1 & 1 & 1 \\
1dkt-A & 1 & 1 & 2 & 32 \\
1fca & 7 & 40 & 36 & 5 \\
1nkl & 1 & 1 & 1 & 1 \\
1trl-A & 56 & 5 & 1 & 4 \\
1pgb & 1 & 1 & 1 & 1 \\
4icb & 1 & 1 & N/A & N/A \\
\hline
\end{tabular}

structures using the discrete state model, we show that these models lead to accurate modeling of native proteins.

The discrete state representation can provide a concise way to represent protein structures by a sequence of states. Unlike representation of secondary structure types (such as $H$ for helices, $E$ for $\beta$ strand, and $C$ for coil and turns), the sequence of discrete states at each residue position uniquely determines the three dimensional conformation. Methods in secondary structure prediction are well developed with prediction accuracy as high as $80 \%$ [67]. Prediction of discrete states can benefit from algorithms developed for secondary structure prediction [68]. Predicted discrete state may be more useful for tertiary structure prediction than predicted secondary structures, since the residue specific discrete states are more informative. Although there have been various attempts to define secondary structure types other than the three basic types, we argue that discrete states provide a natural and flexible representation, where a different number of discrete states can be used for different amino acids. This provides a wide range of models with different complexity and accuracy for studying proteins.

Reduced amino acid residue alphabet. We have simplified amino acid residue alphabet using neighboring residue interactions measured by the first order state transition probability. Hierarchical clustering divides all amino acids into two groups with PRO (proline) and GLY (glycine) separated from the rest of amino acids. Clearly, geometric properties rather than chemical properties dominates at the top level of the clustering. Glycine is very small and the distribution of its $(\alpha, \tau)$ angles has more accessible regions than any other residue types. On the other hand, proline has a very rigid side chain and the distribution of its $(\alpha, \tau)$ angles has 
very limited accessible regions than other amino acid types. These two amino acids are indeed found more frequently in turn/loop conformations among these three secondary structure types ( $\alpha, \beta$, and turns) [69,70]. The remaining amino acids are clustered into two groups with one group being $\{$ ILVYFWCM \} and the other group being $\{$ DNHSTEKAQR , which follows roughly their hydrophobicity at the second level of clustering. This indicates that the local neighboring interaction is also affected significantly by the hydrophobicity of the amino acids [71].

Geometric properties also play important roles in the detailed clustering of amino acids both in the hydrophobic group and the polar group of amino acids. For example, N (Asparagine) and D (Aspartic acid) are clustered together and have large distances to the other polar amino acids. This is quite different from other clustering results based on chemical properties or mutational propensities. This difference is probably due to the fact that both of these amino acids can form favorable intra-residue hydrogen bond between their main chains and the polar group on the side chain. This would affect significantly the geometry of their backbones. Compared with two other similar amino acids, E (Glutamic acid) and Q (Glutamine), D and N are preferred for turn/loop conformations [69,70]. The simplification of amino acids based on local sequencestructure propensities observed in native proteins provides an alternative simplified amino acid alphabet, which will be useful for representation and geometric modeling of protein structures. This simplified alphabet would also be useful in building fragment libraries and in predicting local structures from sequences.

Choice of descriptors. The choice of a specific set of descriptor is critical for the success of potential functions for simplified representations. With a fixed representation, it is always desirable to extract as much useful information as possible by choosing an appropriate set of descriptors. Conversely, since the extractable information from a particular structure are limited by the representation, the consideration of descriptors always affect the choice of representation. Many new descriptors incorporating a variety of different types of information have been developed, such as atomic pair-wise contact calculated by alpha-shape method [15] or Voronoi tessellation [9], distance dependent contact instead of simple distance cut-off $[7,12,72]$, contact order dependent contact [46], and secondary structure dependent contact [73]. We replaced the 210 contact descriptors by distance-dependent contact descriptors with several different distance intervals, but did not observe any noticeable improvement. This is probably because of the limitation of the contact information that can be extracted from simplified representation.

Improving potential function for a fixed representation. Potential functions using weighted linear combination of residue-level contact descriptors defined by simple distance cutoffs have been shown to be inadequate in discriminating many native structures from a large number of decoys $[26,74]$. Among many possible improvements, the modification of descriptors without changing the representation is convenient. Recent study on the conformational biases used in Monte Carlo simulations by several successful folding methods suggest that such conformational biases likely serve as an energy term missing in current potential function [75]. Our work can be regarded as an effort in searching for the missing information of the potential function. In this study, we combine local sequence-structure descriptors with contact descriptors, while keeping both the functional form and representation as simple as possible. Although the current local sequence-structure descriptors are quite simple, the performance of the potential function has been significantly improved compared to the one using only contact propensity.

It is likely that the best potential function will be different if a different protein model is used. Our potential function can be adapted for use with other protein models generated by different sampling methods. The discrimination surface between native proteins and decoys is determined by points (namely, proteins and decoys) along the boundary surface [26]. This surface is determined by the protein model and the method of structure generation, but is invariant once the model and the method are fixed. Therefore, it is necessary to develop different optimized potential functions for different protein models. An improved potential function can be obtained by adding new decoys that are challenging for this particular protein model to the training set. This case-by-case approach is also practical. In applications where a potential 
function is used to discriminate native structures from decoy structures, a researcher usually decides upon choosing a favorite protein model and a structure-generation method, as is the case in research works of protein structure prediction. Since the user has access to a method to generate a large number of candidate structures, decoy structures can be easily obtained for training an improved potential function. This improved potential function can be based on the descriptors and functional form of the original CALSP potential. The effectiveness of this approach can be seen from the performance of our potential functions on LKF and Baker decoy sets, where only decoy structures from LKF and Baker decoy sets are used in training.

Although it is impossible to develop a one-size-fits-all potential function for all simplified models, our study showed that the new set of descriptors, the method for their simplification, and the simple functional form to combine them are generally applicable to other protein models. Our study suggests a novel approach to develop effective potential functions for simplified protein models.

Further improvement of potential function. Potential functions RCALSP1 (using reduced amino acid alphabet in deriving contact descriptors) and RCALSP2 (further incorporating sequence separation information in the descriptors) show slightly better performance compared to CALSP in discrimination of gapless threading decoys, even though they have reduced numbers of parameters, i.e., 455 for RCALSP1 and 565 for RCALSP2, compared to 610 for CALSP. Although more detailed studies are needed to assess the effectiveness of these two potential functions, these results point to a promising direction to further improve the potential function. We expect that many local sequence-structure descriptors are redundant, e.g., some local sequences have no preference for local structure. This indicates that the current set of descriptors can be further simplified, which will provide additional rooms for incorporation of more informative descriptors. Identification of important descriptors will also shed light on the determinants of protein folding and stability.

The local sequence-structure propensity currently used considers only two adjacent residues on the sequence, which cannot capture more complex local interactions beyond the two neighboring residues. Additional descriptors can be derived from two residues not adjacent on the sequence or from more than two residues. The addition of more descriptors will need to be done carefully to avoid the over-fitting problem.

Local sequence-structure relationship and protein folding. Experimental study has shown that the unfolded state of proteins still maintain much of the native topology under strong denaturing condition [76]. The origin of interactions between neighboring residue has been studied recently by electrostatic calculations of peptide solvation [71]. Our results in clustering amino acids based on their sequence neighbor interactions suggests that this mainly originated from the geometric properties of amino acids, but is also significantly influenced by their physicochemical properties. Experimental studies and successful application of local sequence-structure relationship in structure predictions clearly indicate that local sequences or sequence fragments also have strong preference for adopting certain native local structure. Such local sequence-structure relationship could be important for decreasing the large entropy during the folding process. Local sequence-structure correlations induced by neighboring residue interactions may play important roles in the unfolded states, such that the majority of unfolded conformation may be located around the native conformation, although not in the sense of close RMSD [77]. The realization of local and strong sequence-structure correlations may induce more distant but weaker sequence-structure correlations spontaneously at many locations on the peptide sequence, which could be a part of the cooperative folding process. Therefore, the entropy of unfolded state may be dramatically reduced at the very beginning of protein folding due to the correlations between local sequence and structure, which can take effect even during the protein synthesis. The folding entropy, considered as the major force opposing protein folding, therefore may not be so large as thought before.

Summary. Simplified protein representation can effectively reduce the conformational search space and provides an attractive model for computational studies of protein structures. 
However, currently there are no empirical potential functions that are applicable for simplified protein model. In this work, we develop empirical potential function for simplified protein models by combining descriptors derived from residue-residue contact and local sequence-structure relationship. The parameters are obtained by optimizing discrimination of native proteins and decoys. Based on testing with a variety of decoy sets, our results show that this strategy is effective, and the empirical potentials developed here requiring only $C_{\alpha}$ or backbone atoms have better or similar performance in decoy discrimination compared to other residue-level potentials requiring in addition either full atom structure or models of side chains. We also showed that for a large representative set of proteins, discrete state model can be very accurate. We also found that the conformations of nearest sequence neighbors often strongly influence each other, and such correlation can be employed to provide additional discrimination in addition to contact interactions. We further develop reduced alphabet of amino acids based on analysis of local sequence-structure correlation of neighboring residues. The results indicate that there are characteristic properties in adopting local conformations among groups of residues, and such grouping is different from grouping based on contact interactions. We showed that reduced alphabet helps to improve discrimination. The rich information contained in local sequencestructure descriptors suggest that local effects may play important role in reducing entropic cost in protein folding.

Note. Details of the parameters of the potential functions, the angles for the reduced state models of amino acid residues, and a list of the set of 978 proteins can all be found at (gila.bioengr.uic.edu/pub-data/potential05-proteins/).

\section{Acknowledgment}

This work is supported by grants from NSF (CAREER DBI0133856), NIH (GM68958), and ONR (N000140310329).

\section{References}

[1] Anfinsen C. Principles that govern the folding of protein chains. Science 1973;181:223-230.

[2] Dill K. Dominant forces in protein folding. Biochemistry 1990;29:7133-7155.

[3] Dobson C. Protein folding and misfolding. Nature 2003;426:884-890.

[4] Levinthal C. Are there pathways for protein folding? J Chim Phys 1968;65:44-45.

[5] Head-Gordon T, Brown S. Minimalist models for protein folding and design. Curr Opin Struct Biol 2003;13:160-167.

[6] Kolinski A, Skolnick J. Reduced models of proteins and their applications. Polymer 2004; 45:511-524.

[7] Lu H, Skolnick J. A distance-dependent atomic knowledge-based potential for improved protein structure selection. Proteins 2001;44:223-232.

[8] Zhou H, Zhou Y. Distance-scaled, finite ideal-gas reference state improves structure-derived potentials of mean force for structure selection and stability prediction. Protein Science 2002;11:2714-2726.

[9] McConkey B, Sobolev V, Edelman M. Discrimination of native protein structures using atom-atom contact scoring. Proc Natl Acad Sci USA 2003;100:3215-3220.

[10] Bastolla U, Farwer J, Knapp E, Vendruscolo M. How to guarantee optimal stability for most representative structures in the protein data bank. Proteins 2001;44:79-96.

[11] Miyazawa S, Jernigan R. Residue-residue potentials with a favorable contact pair term and an unfavorable high packing density term, for simulation and threading. J Mol Biol 1996; 256:623-644. 
[12] Tobi D, Shafran G, Linial N, Elber R. On the design and analysis of protein folding potentials. Proteins 2000;40:71-85.

[13] Park B, Levitt M. The complexity and accuracy of discrete state models of protein structure. J Mol Biol 1995;249:493-507.

[14] Cline M, Karplus K, Lathrop R, Smith T, Rogers Jr R, Haussler D. Information-theoretical dissection of pairwise contact potentials. Proteins 2002;49:7-14.

[15] Li X, Hu C, Liang J. Simplicial edge representation of protein structures and alpha contact potential with confidence measure. Proteins 2003;53:792-805.

[16] Simons K, Kooperberg C, Huang E, Baker D. Assembly of protein tertiary structures from fragments with similar local sequences using simulated annealing and bayesian scoring functions. J Mol Biol 1997;268:209-225.

[17] Hunter C, Subramaniam S. Protein local structure prediction from sequence. Proteins 2003;pp. 572-579.

[18] Hou Y, Hsu W, Lee M, Bystroff C. Remote homolog detection using local sequence-structure correlations. Proteins 2004;57:518-530.

[19] Shortle D. Composites of local structure propensities: Evidence for local encoding of longrange structure. Protein Science 2002;11:18-26.

[20] Kolodny R, Koehl P, Guibas L, Levitt M. Small libraries of protein fragments model native protein structures accurately. J Mol Biol 2002;323:297-307.

[21] Pei J, Grishin N. Combining evolutionary and structural information for local protein structure prediction. Proteins 2004;56:782-794.

[22] Lezon T, Banavar J, Maritan A. Recognition of coarse-grained protein tertiary structure. Proteins 2004;55:536-547.

[23] Lazaridis T, Karplus M. Effective energy functions for protein structure prediction. Curr Op Struct Biol 2000;10:139-145.

[24] Hao M, Scheraga H. Designing potential energy functions for protein folding. Current Opinion in Structure Biology 1999;9:184-188.

[25] Buchete N, Straub J, Thirumalai D. Development of novel statistical potentials for protein fold recognition. Curr Opin Struct Biol 2004;14:225-232.

[26] Hu C, Li X, Liang J. Developing optimal nonlinear scoring function for protein design. Bioinformatics 2004;20:3080-3098.

[27] Thomas P, Dill K. Statistical potentials extracted from protein structures: How accurate are they? J Mol Biol 1996;257:457-469.

[28] Park B, Levitt M. Energy functions that discriminate x-ray and near-native folds from well-constructed decoys. J Mol Biol 1996;258:367-392.

[29] Wang G, Dunbrack RLJ. PISCES: a protein sequence culling server. Bioinformatics 2003; 19:1589-1591.

[30] Adamian L, Jackups Jr R, Binkowski A, Liang J. Higher order interhelical spatial interactions in membrane proteins. J Mol Biol 2003;327:251-272.

[31] Fain B, Xia Y, Levitt M. Design of an optimal Chebyshev-expanded discrimination function for globular proteins. Protein Sci 2002;11:2010-2021.

[32] Riddle D, Santiago J, Bray-Hall S, Doshi N, Grantcharova V, Yi Q, Baker D. Functional rapidly folding proteins from simplified amino acid sequences. Nat Struct Biol 1997;4:805809.

[33] Li T, Fan K, Wang J, Wang W. Reduction of protein sequence complexity by residue grouping. Protein Eng 2003;16:323-330. 
[34] Cannata N, Toppo S, Romualdi C, Valle G. Simplifying amino acid alphabet by mean of a branch and bound algorithm and substitution matrices. Bioinformatics 2002;18:1102-1108.

[35] Murphy LR, Wallqvist A, Levy R. Simplified amino acid alphabets for protein fold recognition and implications for folding. Protein Engineering 2000;13:149-152.

[36] Wang J, Wang W. A computational approach to simplifying the protein folding alphabet. Nature Structural Biology 1999;6:1033-1038.

[37] Dill K. Theory for the folding and stability of globular proteins. Biochemistry 1985;24:1501.

[38] Sagot M, Viari A, Soldano H. Multiple sequence comparison - a peptide matching approach. Theor Comp Sci 1997;180:115-137.

[39] Gan H, Tropsha A, Schlick T. Generatin folded protein structures with a lattice growth algorithm. J Chem Phys 2000;113:5511-5524.

[40] Liang J, Zhang J, Chen R. Statistical geometry of packing defects of lattice chain polymer from enumeration and sequential Monte Carlo method. J Chem Phys 2002;117:3511-3521.

[41] Zhang J, Liu J. A new sequential importance sampling method and its application to the two-dimensional hydrophobic-hydrophilic model. J Chem Phys 2002;117:3492-3498.

[42] Zhang J, Chen R, Tang C, Liang J. Origin of scaling behavior of protein packing density: A sequential Monte Carlo study of compact long chain polymers. J Chem Phys 2003; 118:6102-6109.

[43] Zhang J, Chen Y, Chen R, Liang J. Importance of chirality and reduced flexibility of protein side chains: a study with square and tetrahedral lattice models. J Chem Phys 2004;121:592-603.

[44] Brooks BR, Bruccoleri RE, Olafson BD, States DJ, Swaminathan S, Karplus M. CHARMM: A program for macromolecular energy, minimization, and dynamics calculations. J Comp Chem 1983;4:187-217.

[45] Pearlman D, Case D, Caldwell J, Ross W, Cheatham T, DeBolt S, Ferguson D, Seibel G, Kollman P. AMBER, a computer program for applying molecular mechanics, normal mode analysis, molecular dynamics and free energy calculations to elucidate the structures and energies of molecules. Comp Phys Commun 1995;91:1-41.

[46] Betancourt M. A reduced protein model with accurate native-structure identification ability. Proteins 2003;53:889-907.

[47] Tobi D, Shafran G, Linial N, Elber R. On the design and analysis of protein folding potentials. Proteins 2000;40:71-85.

[48] Chiu T, Goldstein R. Optimizing energy potentials for success in protein tertiary structure prediction. Folding Des 1998;3:223-228.

[49] Mirny L, Shakhnovich E. How to derive a protein folding potential? A new approach to an old problem. J Mol Biol 1996;264:1164-1179.

[50] Vendruscolo M, Domany E. Pairwise contact potentials are unsuitable for protein folding. J Chem Phys 1998;109:11101-11108.

[51] Dima R, Banavar J, Maritan A. Scoring functions in protein folding and design. Protein Sci 2000;9:812-819.

[52] Goldstein R, Luthey-Schulten Z, Wolynes P. Protein tertiary structure recognition using optimized hamiltonians with local interactions. Proc Natl Acad Sci USA 1992;89:90299033.

[53] Friedrichs M, Wolynes P. Toward protein tertiary structure recognition by means of associative memory hamiltonians. Science 1989;246:371-373.

[54] Schölkopf B, Smola A. Learning with kernels: Support vector machines, regularization, optimization, and beyond. Cambridge, MA: The MIT Press, 2002. 
[55] Joachims T. Making large-scale support vector machine learning practical. In: Scholkopf B, C. B, A. S, editors, Advances in Kernel Methods: Support Vector Machines, MIT Press, Cambridge, MA. 1998; .

[56] Maiorov V, Crippen G. Contact potential that recognizes the correct folding of globular proteins. J Mol Biol 1992;227:876-888.

[57] Loose C, Klepeis J, Floudas C. A new pairwise folding potential based on improved decoy generation and side-chain packing. Proteins 2004;54:303-314.

[58] Guntert P, Mumenthaler C. Torsion angle dynamics for NMR structure calculation with the new program DYANA. JMol Biol 1997;273:283-298.

[59] Simons K, Bonneau R, Ruczinski I, Baker D. Ab initio protein structure prediction of CASP III targets using ROSETTA. Proteins 1999;3:171-176.

[60] Tsai J, Bonneau R, Morozov A, Kuhlman B, Rohl C, Baker D. An improved protein decoy set for testing energy functions for ptoein structure prediction. Proteins 2003;53:76-87.

[61] Samudrala R, Levitt M. Decoys 'R' us: a database of incorrect conformations to improve protein structure prediction. Protein Science 2000;9:1399-1401.

[62] Samudrala R, Xia Y, Levitt M, Huang E. A combined approach for ab initio construction of low resolution protein tertiary structures from sequence. Pac Symp Biocomput 1999;.

[63] Xia Y, Levitt M. Extracting knowledge-based energy functions from protein structures by error rate minimization: Comparison of methods using lattice model. J Chem Phys 2000; 113:9318-9330.

[64] Fletcher R. A new approach to variable metric algorithms. Comput J 1970;13:317-322.

[65] Vriend G, Sander C. Quality control of protein models - directional atomic contact analysis. J Appl Cryst 1993;26:47-60.

[66] Levitt M. Molecular dynamics of native protein: I. computer simulation of trajectories. J Mol Biol 1983;168:595-620.

[67] McGuffin L, Bryson K, Jones D. The PSIPRED protein structure prediction server. Bioinformatics 2000;16:404-405.

[68] Kuang R, Leslie C, Yang A. Protein backbone angle prediction with machine learning approaches. Bioinformatics 2004;20:1612-1621.

[69] Crasto CJ, Feng J. Sequence code for extended conformation: a neighbor-dependent sequence analysis of loops in proteins. Proteins 2001;42:399-413.

[70] Xia X, Xie Z. Protein structure, neighbor effect, and a new index of amino acid dissimilarities. Mol Biol Evol 2002;19:58-67.

[71] Avbelj F, Baldwin R. Origin of the neighboring residue effect on peptide backbone conformation. Proc Natl Acad Sci USA 2004;101:10967-10972.

[72] Bahar I, R.L. J. Inter-residue potentials in globular proteins and the dominance of highly specific hydrophilic interactions at close separation. J Mol Biol 1997;226:195-214.

[73] Simons K, Ruczinski I, Kooperberg C, Fox B, Bystroff C, Baker D. Improved recognition of native-like protein structures using a combination of sequence-dependent and sequenceindependent features of proteins. Proteins 1999;34:82-95.

[74] Vendruscolo M, Najmanovich R, Domany E. Can a pairwise contact potential stabilize native protein folds against decoys obtained by threading? Proteins 2000;38:134-148.

[75] Przytycka T. Significance of conformational biases in Monte Carlo simulations of protein folding: Lessons from Metropolis-Hastings approach. Proteins 2004;57:338-344.

[76] Shortle D, Ackerman M. Persistence of native-like topology in a denatured protein in $8 \mathrm{~m}$ urea. Science 2001;293:487-489.

[77] Fitzkee N, Rose G. Reassessing random-coil statistics in unfolded proteins. Proc Natl Acad Sci USA 2004;101:12497-12502. 